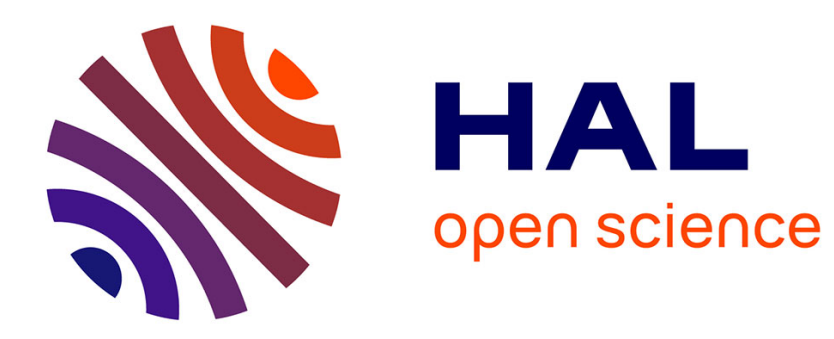

\title{
Mathematical modeling of the LCD response time
}

Pierre Adam, Pascal Bertolino, Fritz Lebowsky

\section{To cite this version:}

Pierre Adam, Pascal Bertolino, Fritz Lebowsky. Mathematical modeling of the LCD response time. Journal of the Society for Information Display, 2007, 15 (8), pp.571-577. 10.1889/1.2770857 . hal00177263

\section{HAL Id: hal-00177263 \\ https://hal.science/hal-00177263}

Submitted on 6 Oct 2007

HAL is a multi-disciplinary open access archive for the deposit and dissemination of scientific research documents, whether they are published or not. The documents may come from teaching and research institutions in France or abroad, or from public or private research centers.
L'archive ouverte pluridisciplinaire HAL, est destinée au dépôt et à la diffusion de documents scientifiques de niveau recherche, publiés ou non, émanant des établissements d'enseignement et de recherche français ou étrangers, des laboratoires publics ou privés. 


\title{
Mathematical modeling of the $L C D$ response time
}

\author{
Pierre Adam 1,2, Pascal Bertolino 1, Fritz Lebowsky 2 \\ 1 GIPSA-lab, INPG-CNRS, Grenoble, France, email: pierre.adam@lis.inpg.fr, \\ pascal.bertolino@inpg.fr \\ 2 STMicroelectronics, 12 rue Jules Horowitz BP 217, 38019 Grenoble, France, \\ email: fritz.lebowsky@st.com
}

\begin{abstract}
Techniques to reduce LCD motion blur are extensively used in industry and they depend on an inherent LCD parameter: response time. However, normative response time is not a sufficient reference to improve LCD performance and all the gray-to-gray response time quantities are required to obtain a good improvement quality. However, measuring and gathering all the gray-to-gray transitions takes an excessive time measurement. Consequently, we propose a novel LCD model to simulate as well as compute grayto-gray transitions (response time and behavior) from a reduced measurement set in order to decrease the response time measurement.
\end{abstract}

Keywords: Liquid crystal display, response time model, curve fitting,

\section{Introduction}

Recently, active matrix liquid crystal display acceptance has considerably increased for PC monitors and TVs. TFT-LCDs carry the advantage of flatness, weight, low power consumption and high resolution but also, share an inherent weakness, motion blur due to a hold type driving method and response time of liquid crystal cells. This previous defect produces tailing phenomena and motion blur, easily detected as a visual artefact [1].

For many years, reducing LCD response time and motion blur has been an industry focus to improve display image quality. Many solutions were proposed such as black insertion [2], blinking backlight [3], double frame-rate [4], motion compensated inverse filtering [5] and the widely used technique introduced in 1992, called overdrive [6].

However, speeding-up the LC pixels response time with current overdrive methods needs a Look-Up-Table (L.U.T.) which contains correction data. These values are obtained with measurements of original liquid crystal cell response time with a specific measurement system [7]. Figure 1 shows an example of gray-to-gray response time for rising transitions (from gray levels to lighter ones), obtained from a 19-inch Twisted Nematic LCD monitor.

A classical approach with basic process to correctly fill a L.U.T. takes an excessive time. However, some promising efforts recently appeared [8-9] to decrease the computation time.

For instance, a $32 \times 32$ Look-up-Table needs at least
496 response time measurements for rising transitions to be correctly filled. Furthermore, it is necessary to make several measurements for each desired response time in order to obtain an accurate mean value.

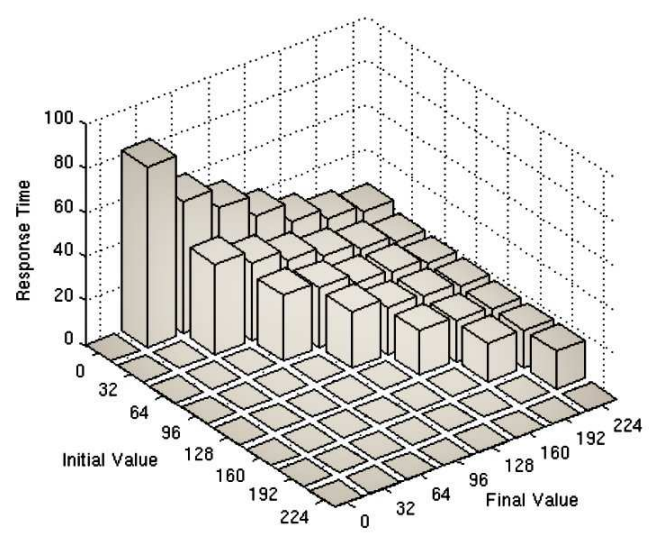

Figure 1: Discrete gray to gray response time of the 19inch monitor panel for rising transitions.

However, this amount of measurements can be drastically reduced. Therefore, we introduce a new approach to radically simplify the gathering of response time data by modelling the behaviour of LC cells transition and by estimating gray to gray response times.

In this paper, we first define some mathematical sets and functions used for the model of response time estimation. Then, we split our model in two parts: transitions with zero initial gray level and non-zero initial gray level. Finally, we compute the associated response time for both models and we evaluate their relevancy compared to actual data acquired from a 19inch Twisted-Nematic LCD monitor with a response time measurement system.

\section{Response time definition}

In this section, we define some sets and subsets for mathematically describing the gray level transitions and the associated response time.

Let $\mathbf{L}_{\mathbf{n}}=\left\{L_{i} / 0 \leq i<n, L_{i}=i\right\}$ be a set containing $n$ integers representing initial and final gray level values of transitions. For example, with 8 bit coded colour components, $L_{256}=[0 . .255]$ and the total of gray level equals 256.

Define $\mathbf{T}$ as another set generated by $\mathbf{L}_{n}$ and 
representing gray level transitions from $\mathbf{L}_{n}$ to $\mathbf{L}_{n} ; \mathbf{T}$ is a set of integer pairs as described in equation 1 :

$\mathbf{T}=\left\{(x, y) / x \in \mathbf{L}_{\mathbf{n}}, y \in \mathbf{L}_{\mathbf{n}}, x \neq y\right\}$

where $x$ and $y$ represent respectively the initial and the final gray level of the transition. The number of gray level transitions equals to $n^{2}-n$ with $n$ the number of different gray level. Actually we do not consider $(x, x)$ as a transition. Moreover, the order of the elements is primordial: $(x, y)$ is different from $(y, x)$, and stand for two opposite transitions.

Define two subsets $\downarrow \mathbf{T}$ and $\uparrow \mathbf{T}$ from the set $\mathbf{T}$ : the first subset represents the falling transitions (from a gray level to an inferior one) and the other one the rising transitions (from a gray level to a superior one) by the Eq. (2) and Eq. (3):

$\downarrow \mathbf{T}=\{(x, y) /(x, y) \in \mathbf{T}, x>y\}$
$\uparrow \mathbf{T}=\{(x, y) /(x, y) \in \mathbf{T}, x<y\}$

We notice the two subset have the same size ( $\left.\frac{n^{2}-n}{2}\right)$ and the union of $\downarrow \mathbf{T}$ and $\uparrow \mathbf{T}$ entirely covers the set $\mathbf{T}$ : each transition is either a rising transition or a falling transition. Moreover, if (x,y) is in $\downarrow \mathbf{T}$, then its opposite $(\mathrm{y}, \mathrm{x})$ is in $\uparrow \mathbf{T}$.

Concerning our model presented in this article, only rising transitions (elements of $\uparrow \mathbf{T}$ ) will be estimated.

Split $\uparrow \mathbf{T}$ into two subsets: $\uparrow \mathbf{T}_{X}$ the subset of rising transitions with a fixed initial value equal to $X$ and $\uparrow \mathbf{T}^{Y}$, the subset of rising transitions with a fixed final value equal to Y. So, we have the Eq. (4) and (5):

$$
\begin{aligned}
& \uparrow \mathbf{T}_{X}=\{(x, y) /(x, y) \in \uparrow \mathbf{T}, x=X\} \\
& \uparrow \mathbf{T}^{Y}=\{(x, y) /(x, y) \in \uparrow \mathbf{T}, y=Y\}
\end{aligned}
$$

The number of elements of $\uparrow \mathbf{T}_{X}$ equals $(n-1-X)$ elements while the $\uparrow \mathbf{T}^{Y}$ subset only contains $\mathrm{Y}$ elements.

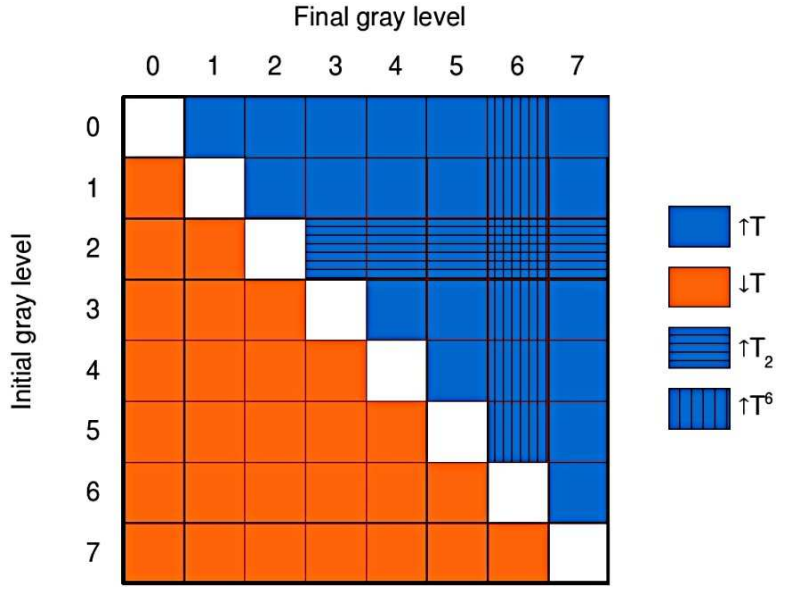

Figure 2: Matrix representation of $\downarrow \mathbf{T}, \uparrow \mathbf{T}, \uparrow \mathbf{T}_{2}$ and $\uparrow \mathbf{T}^{6}$ generated by $\mathbf{L}_{8}$ (8 gray levels).
We define two particular subsets $\uparrow \mathbf{T}_{0}$ and $\uparrow \mathbf{T}_{*}$ : $\uparrow \mathbf{T}_{0}$ represents the zero initial gray level transitions while $\uparrow \mathbf{T}_{*}$ represents the non-zero initial gray level transitions.

Figure 2 depicts a matrix representation of the different sets and subsets presented in this section with 8 gray levels.

Define $L_{i}$ and $L_{f}$ respectively as the values of an initial gray level and a final gray level. Finally, define $L_{L_{i}}^{L_{f}}$ as the transition from initial gray level $L_{i}$ to final gray level $L_{f}$.

Let $C(t)$ be a bounded and strictly monotonic function from $\left[t_{0} ; \infty\right]$ to $\left[L_{i} ; L_{f}\right] . C(t)$ represents the behaviour of the response time. Define $t_{10}$ and $t_{90}$ as the time instant when $C(t)$ reaches $10 \%$ and $90 \%$ of the final value, as we can see on Eq. (6) and (7):

$$
\begin{aligned}
& C\left(t_{10}\right)=0.1 \times\left(L_{f}-L_{i}\right)+L_{i} \\
& C\left(t_{90}\right)=0.9 \times\left(L_{f}-L_{i}\right)+L_{i}
\end{aligned}
$$

The response time of the curve $C(t)$, denoted $\tau$, is then computed in compliance with the VESA FPDM standard [9]:

$\tau=t_{90}-t_{10}$

Figure 3 depicts the relationship between the transition curve $C(t)$ and the associated response time $\tau$, shown on Eq. (6), (7) and (8).

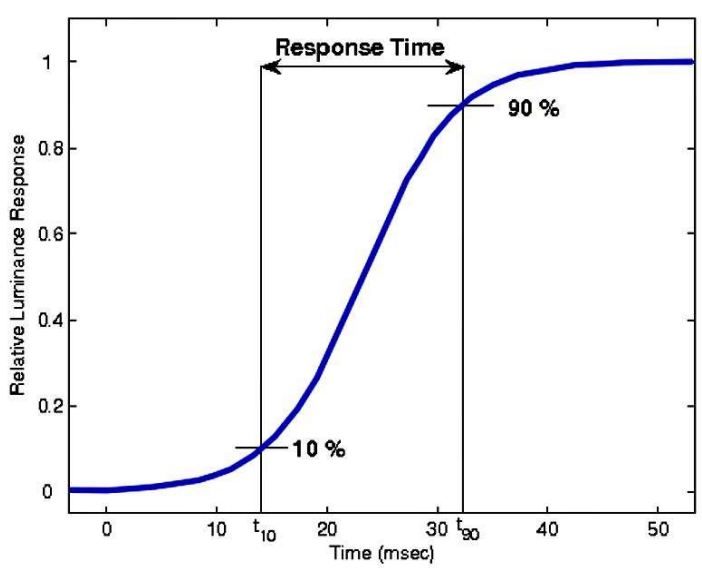

Figure 3: VESA response time computation.

\section{Model of transition with zero initial value}

In this section, we begin to construct our model with the subset $\uparrow \mathbf{T}_{0}$ of rising transitions of which initial gray level is zero, by estimating the response time and then the $\mathrm{LC}$ behaviour.

\subsection{Response time}


As shown in Figure 1, the response time of $\uparrow \mathbf{T}_{0}$ is a decreasing function of the final gray level. To avoid taking too many measurements on a display panel, we estimate on Eq. (9) the behaviour of the response time as an $n^{\text {th }}$ order polynomial function of the final gray level.

$\tau=\sum_{k=0}^{n} a_{k} \cdot\left(L_{f}\right)^{k}$

where $\left(a_{k}\right)_{0 \leq k \leq n}$ are denoted as unknown constants to be found, $\tau$ the measured response time and $L_{f}$ the value of the final gray level of the transition $L_{0}^{L_{f}}$.

A polynomial function was chosen because of its possibility to correctly fit with any type of response time behaviour. To find the parameters $\left(a_{k}\right)_{0 \leq k \leq n}$, we create a system of $m$ polynomial functions (Eq. 10) which needs at least $n+1$ different measurements (consequently, we have $m \geq n+1$ ); the $\mathrm{i}^{\text {th }}$ measurement represents the final gray level $L_{f}(i)$ and its associated response time $\tau(i)$.

$$
\left\{\begin{array}{c}
\tau(1)=\sum_{k=0}^{n} a_{k} \cdot\left(L_{f}(1)\right)^{k} \\
\tau(2)=\sum_{k=0}^{n} a_{k} \cdot\left(L_{f}(2)\right)^{k} \\
\ldots \ldots \\
\tau(m)=\sum_{k=0}^{n} a_{k} \cdot\left(L_{f}(m)\right)^{k}
\end{array}\right.
$$

Define $\boldsymbol{\Gamma}=(\tau(i))_{1 \leq i \leq m}$, a vector of the measured response time and $\boldsymbol{\Lambda}=\left(L_{f}(i)\right)_{1 \leq i \leq m}$ a vector of associated final gray level. The size of $\boldsymbol{\Gamma}$ and $\boldsymbol{\Lambda}$ equals to $m$. Therefore, the previous system can be written as the vector equation.

$$
\boldsymbol{\Gamma}=\sum_{k=0}^{n} a_{k} \cdot(\boldsymbol{\Lambda})^{k}
$$

If $m=n+1$, Eq. (11) describes a theoretical solution of our problem with no possible error: since we have $m$ equations with $m$ unknown constants, the model correctly fits the measured values and not necessarily the others. Consequently, there will be errors on the estimated response times.

With $m>n+1$, we have $m$ equations with $n$ unknown constants. Consequently, these additional values fit all the response time but add a slight systematic error $r(i)$ on each equation.

Therefore, we introduce the vector $\mathbf{R}=(r(i))_{1 \leq i \leq m}$ the set of systematic errors which must be minimized and thereby Eq. (11) becomes Eq. (12).
$\boldsymbol{\Gamma}+\mathbf{R}=\sum_{k=0}^{n} a_{k} \cdot(\mathbf{\Lambda})^{k}$

The more the amount of measurements increases, the more systematic errors $r(i)$ decrease. In order to find the unknown constants $\left(a_{k}\right)_{0 \leq k \leq n}$, we must minimize the quadratic error, denoted $\mathbf{E}$.

$\mathbf{E}=\mathbf{R} \cdot \mathbf{R}$

$\mathbf{E}=\left(\sum_{k=0}^{n} a_{k} \cdot(\boldsymbol{\Lambda})^{k}-\boldsymbol{\Gamma}\right) \cdot\left(\sum_{k=0}^{n} a_{k} \cdot(\boldsymbol{\Lambda})^{k}-\boldsymbol{\Gamma}\right)$

$\mathbf{E}$ reaches its minimum value when all its partial derivative reaches 0 . Therefore, by deriving Eq. (14), we obtain $n$ partial equations as Eq. (15).

$\frac{d \mathbf{E}}{d a_{i}}=2 \times \boldsymbol{\Lambda}^{i} \times \sum_{k=0}^{n} a_{k} \cdot(\mathbf{\Lambda})^{k}-2 \times \mathbf{\Lambda}^{i} \times \boldsymbol{\Gamma}$

Define a matrix $\mathbf{M}=\left(m_{i j}\right)_{0 \leq i, j \leq n}$ with the coefficient $m_{i j}=\boldsymbol{\Lambda}^{i} \cdot \boldsymbol{\Lambda}^{j}$, the vector $\mathbf{B}=\left(b_{i}\right)_{0 \leq i \leq n}$ with $b_{i}=\boldsymbol{\Lambda}^{i} \times \boldsymbol{\Gamma}$ and the vector $\mathbf{A}=\left(a_{i}\right)_{0 \leq i \leq n}$ with $\left(a_{i}\right)_{0 \leq i \leq n}$ the unknown constants to be found. The previous problem of minimization of the equation equals to solve the matrix equation $\mathbf{M}=\mathbf{A} \times \mathbf{B}$. Finally, we obtain the parameters with $\mathbf{A}=\mathbf{M}^{-1} \times \mathbf{B}$.

Figure 4 shows an estimation of response time with a third order polynomial function initialized with 7 measurements on a Twisted Nematic LCD monitor.

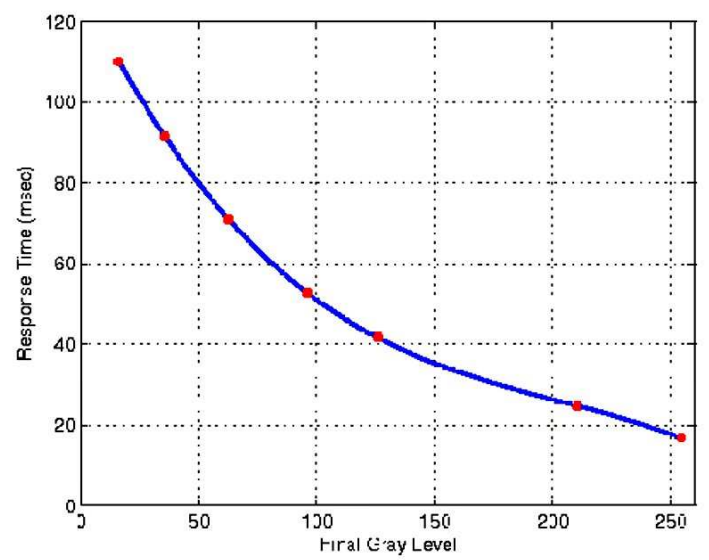

Figure 4: Estimation of response time for rising transitions in $\uparrow \mathbf{T}_{0}$ with polynomial approach (blue) initialized by 7 measurements (red).

\subsection{LC behaviour}

The next step is to model the transition as a temporal evolution of the gray level. We can see an example of the behaviour of $L_{0}^{96}$ illustrated in Figure 5.

With a general approach to keep the two horizontal asymptotes and the general behaviour of the curve, a hyperbolic tangent model is proposed. Physically, this 
model aims at representing the switching on of the LCD cell.

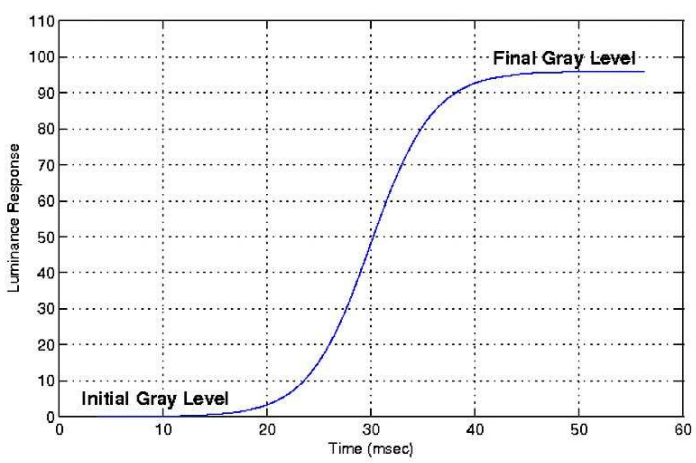

Figure 5: Example of transition: $L_{0}^{96}$.

To correctly fit the model to the actual behaviour of all LC transitions, our function needs three parameters: the response time $(\tau)$ which lets the transition's velocity evolve, the final gray level $\left(L_{f}\right)$ for setting the

final vertical asymptotic value and a temporal shift $\left(t_{I}\right)$ which centres the model in the correct temporal window. Until the end of the article, the simulated response time (presented in the previous subsection) will be used in this function instead of measured values.

Therefore, the LC's behaviour can be described by the function $C(t)$ with equation 16 :

$C(t)=\left(1+\tanh \left(\frac{2 \cdot \operatorname{atanh}(0.8) \cdot\left(t-t_{I}\right)}{\tau}\right)\right) \cdot \frac{L_{f}}{2}$

We notice that this function, that represents a transition between 0 and $L_{f}$, is bounded between these two values. Eq (17) and (18) show the theoretical (and above mathematical) computation of the limits of $C(t)$.

$$
\begin{aligned}
& \lim _{t \rightarrow-\infty} C(t)=\lim _{t \rightarrow-\infty}(1+\tanh (t)) \times \frac{L_{f}}{2}=0 \\
& \lim _{t \rightarrow+\infty} C(t)=\lim _{t \rightarrow+\infty}(1+\tanh (t)) \times \frac{L_{f}}{2}=L_{f}
\end{aligned}
$$

Moreover, the response time computation formula (Eq. (6) and (7)), presented in section 2, applied on the curve $C(t)$ allows extracting the simulated response time value. We first compute $C\left(t_{10}\right)$ and $C\left(t_{90}\right)$.

$$
\left\{\begin{array}{l}
C\left(t_{10}\right)=\left(1+\tanh \left(\frac{2 \cdot \operatorname{atanh}(0 \cdot 8) \cdot\left(t_{10}-t_{I}\right)}{\tau}\right)\right) \cdot \frac{L_{f}}{2} \\
C\left(t_{90}\right)=\left(1+\tanh \left(\frac{2 \cdot \operatorname{atanh}(0 \cdot 8) \cdot\left(t_{90}-t_{I}\right)}{\tau}\right)\right) \cdot \frac{L_{f}}{2}
\end{array}\right.
$$

Furthermore, with the definition of the response time, Eq. (6) and (7) and the fact that $L_{i}=0$, we also have:

$$
\left\{\begin{array}{l}
C\left(t_{10}\right)=0.1 \times L_{f} \\
C\left(t_{90}\right)=0.9 \times L_{f}
\end{array}\right.
$$

Consequently, by combining Eq. (19) and (20), we can easily compute the difference $t_{90}-t_{10}$ by:

$$
\begin{aligned}
& \left\{\begin{array}{l}
\tanh \left(\frac{2 \cdot \operatorname{atanh}(0.8) \cdot\left(t_{10}-t_{I}\right)}{\tau}\right)=-0.8 \\
\tanh \left(\frac{2 \cdot \operatorname{atanh}(0.8) \cdot\left(t_{90}-t_{I}\right)}{\tau}\right)=0.8
\end{array}\right. \\
& \left\{\begin{array}{l}
2 . \operatorname{atanh}(0.8) \cdot\left(t_{10}-t_{I}\right)=-\tau \times \operatorname{atanh}(0.8) \\
2 . \operatorname{atanh}(0.8) \cdot\left(t_{90}-t_{I}\right)=\tau \times \operatorname{atanh}(0.8)
\end{array}\right. \\
& \left\{\begin{array}{c}
2 .\left(t_{10}-t_{I}\right)=-\tau \\
2 .\left(t_{90}-t_{I}\right)=\tau
\end{array}\right.
\end{aligned}
$$

Finally, with the difference of the two formulas of Eq. (23), we find the expected result $t_{90}-t_{10}=\tau$. Therefore, the LC behaviour model is in compliance with the VESA response time definition.

\section{Model extension to other transitions}

After modelling the transitions of $\uparrow \mathbf{T}_{0}$ (in section 3 ), we extend the model to $\uparrow \mathbf{T}_{*}$. Figure 6 shows the superposition of the actual measurements: one transition from $\uparrow \mathbf{T}_{0}\left(L_{0}^{128}\right)$ and three transitions from $\uparrow \mathbf{T}_{*}$ $\left(L_{32}^{128}, L_{64}^{128}, L_{96}^{128}\right.$ ).

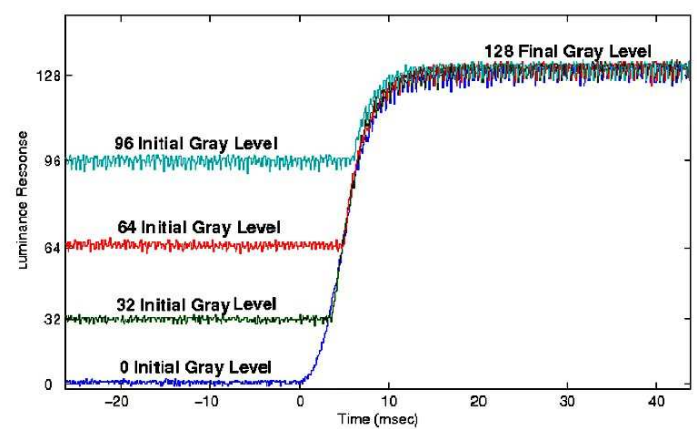

Figure 6: Superposition of measured transitions $L_{32}^{128}$,

$$
L_{64}^{128} \text { and } L_{96}^{128} \text { over } L_{0}^{128} \text {. }
$$

According to this relationship, we can deduce the LC behaviour of any transitions of $\uparrow \mathbf{T}^{Y}$ (with $Y \neq 0$ ), from the curve of the transition $L_{0}^{Y}$.

Define $C(t)$ the curve of $L_{0}^{L_{f}}$ (in $\uparrow \mathbf{T}_{0}$ ) and $D(t)$ the curve of $L_{L_{i}}^{L_{f}}$ (in $\uparrow \mathbf{T}^{L_{f}}$ ). Define $t_{10}$ and $t_{90}$ as the time instant when $C(t)$ reaches $10 \%$ and $90 \%$ of the final value. Define $t^{\prime}{ }_{10}$ and $t^{\prime}{ }_{90}$ as the time instant when $D(t)$ reaches $10 \%$ and $90 \%$ of the final value.

Consistent with Figure $6, D\left(t^{\prime}{ }_{10}\right)$ and $D\left(t^{\prime}{ }_{90}\right)$ are not in the constant part of $D(t)$ (i.e. the part equals to $\left.L_{i}\right)$ and these two points are part of the curve $C(t)$. Consequently, we can write the following relationship: 
$\left\{\begin{array}{l}D\left(t^{\prime}{ }_{10}\right)=C\left(t^{\prime}{ }_{10}\right) \\ D\left(t^{\prime}{ }_{90}\right)=C\left(t^{\prime}{ }_{90}\right)\end{array}\right.$

Thanks to these formulas, we can now compute the new response time of the transition $L_{L_{i}}^{L_{f}}$ denoted $\tau^{\prime}$ from the value of the response time of $L_{0}^{L_{f}}$ denoted $\tau$. Based on Eq. (16), we first compute $C\left(t^{\prime}{ }_{10}\right)$ and $C\left(t^{\prime}{ }_{90}\right)$.

$$
\left\{\begin{array}{l}
C\left(t^{\prime}{ }_{10}\right)=\left(1+\tanh \left(\frac{2 \cdot \operatorname{atanh}(0 \cdot 8) \cdot\left(t_{10}^{\prime}-t_{I}\right)}{\tau}\right)\right) \cdot \frac{L_{f}}{2} \\
C\left(t^{\prime}{ }_{90}\right)=\left(1+\tanh \left(\frac{2 \cdot \operatorname{atanh}(0 \cdot 8) \cdot\left(t_{90}^{\prime}-t_{I}\right)}{\tau}\right)\right) \cdot \frac{L_{f}}{2}
\end{array}\right.
$$

Furthermore, with the definition of the response time of $D(t)$ and Eq. (6) and Eq. (7), we have also the relationship:

$$
\left\{\begin{array}{c}
D\left(t^{\prime}{ }_{10}\right)=0.1 \times\left(L_{f}-L_{i}\right)+L_{i}=C\left(t^{\prime}{ }_{10}\right) \\
D\left(t^{\prime}{ }_{90}\right)=0.9 \times\left(L_{f}-L_{i}\right)+L_{i}=C\left(t^{\prime}{ }_{90}\right)
\end{array}\right.
$$

Consequently, we can easily compute the difference $t^{\prime}{ }_{90}-t^{\prime}{ }_{10}=\tau^{\prime}$ by combining Eq. (25) and Eq. (26) in Eq. (27).

$$
\left\{\begin{array}{l}
\tanh \left(\frac{2 \cdot \operatorname{atanh}(0.8) \cdot\left(t^{\prime} 10^{-t} I\right)}{\tau}\right)=-0.8+1.8 p \\
\tanh \left(\frac{2 \cdot \operatorname{atanh}(0.8) \cdot\left(t^{\prime} 90^{-t} I\right)}{\tau}\right)=0.8+0.2 p
\end{array}\right.
$$

with $p=\frac{L_{i}}{L_{f}}$, the ratio between the initial level and the final level of the transition. We apply the inverse hyperbolic tangent function to Eq. (27) and compute the relationship on $\mathrm{Eq}(6)$.

$$
\begin{aligned}
& \left\{\begin{array}{c}
2 . \operatorname{atanh}(0.8) \cdot\left(t^{\prime}{ }_{10}-t_{I}\right)=\tau \times \operatorname{atanh}(-0.8+1.8 p) \\
\text { 2. } \operatorname{atanh}(0.8) \cdot\left(t^{\prime}{ }_{90}-t_{I}\right)=\tau \times \operatorname{atanh}(0.8+0.2 p)
\end{array}\right. \\
& \tau^{\prime}=\tau .\left(\frac{\operatorname{atanh}(0.8+0.2 p)-\operatorname{atanh}(-0.8+1.8 p)}{2 \cdot \operatorname{atanh}(0.8)}\right)
\end{aligned}
$$

Finally, we simplify the previous equation with the following equivalence (with $-1<x<1$ ):

$$
\operatorname{atanh}(x)=\frac{1}{2} \ln \left(\frac{1+x}{1-x}\right)
$$

We obtain the next equation that links $\tau^{\prime}$ the response time of $L_{L_{i}}^{L_{f}}$ to $\tau$ the response time of $L_{0}^{L_{f}}$ :

$$
\tau^{\prime}=\frac{\tau}{4 . \ln (3)} \ln \left(1+\frac{16}{1.8 p+0.2}\right)
$$

$$
\tau^{\prime}=K(p) \times \tau
$$

Figure 7 shows the evolution of the function $K(p)$ with $0<p<1$. As we can notice it in this figure and in Eq. 31, when $p=0, L_{i}=0$ and $K(p)=1$ and we get back the expected value $\tau^{\prime}=\tau$.

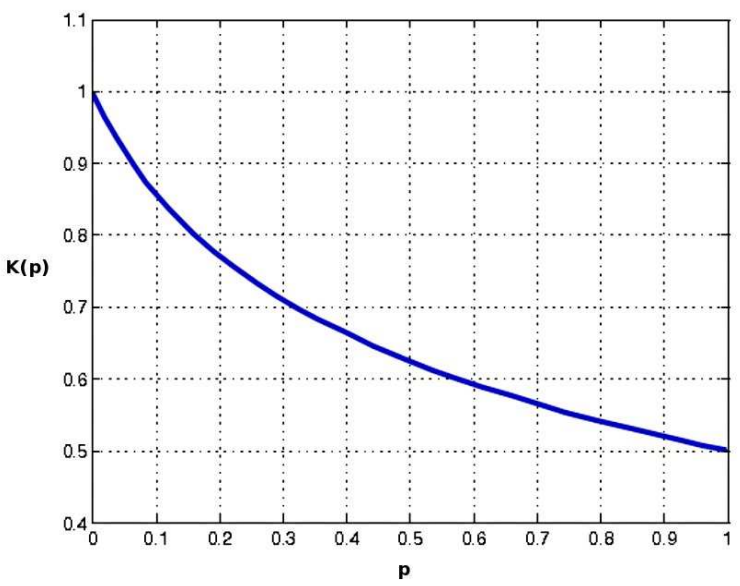

Figure 7: Evolution of $K(p)$ with $0<p<1$.

With respect to Eq. (1) and Eq. (3), $L_{i}$ cannot be equal to $L_{f}$. Nevertheless, when $L_{i}$ converges to $L_{f}$, p converges to 1 and the new response time $\tau^{\prime}$ will converge towards $\frac{\tau}{2}$ instead of the commonly expected value 0 .

This result is due to our hyperbolic tangent model which never reaches its asymptote (the transition's final level). So, when $\mathrm{p}$ is close to 1 , we have to consider the response time estimation as a theoretical value instead of an actual result.

\section{Results}

Tests were carried out on a 19-inch TN LCD monitor without any enhancement algorithm such as overdrive. The model of the response time from $\uparrow \mathbf{T}_{0}$ transitions has been initialized with 7 measurements performed with a measurement system based on a high speed camera.

Figure 8 compares the LC behaviour on the transition $L_{0}^{96}$ with our hyperbolic tangent model $C(t)$ and a measured transition curve $M(t)$.

The main parameter of the model (i.e. the response time $\tau$ ) has been simulated with a third order polynomial function initialized with the 7 measurements. The relative error between the simulated response time and the real measure is close to $5 \%$. 


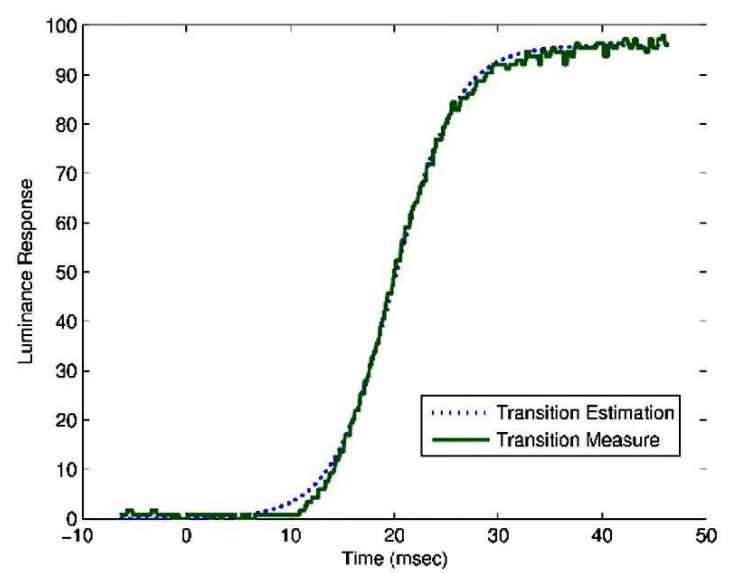

Figure 8: Comparison between an actual measurement of $L_{0}^{96}$ and the simulated transition (with estimated response time)

First, with the estimated response time of $L_{0}^{96}$, we compute the absolute error $E_{a}$ between our model and the real behaviour of the transition.

$$
E_{a}=\max _{t}(|C(t)-M(t)|)
$$

We obtain a maximum absolute error close to 3 gray levels, located in small gray level. Then, we compute the mean relative error $E_{r}$ between our model and the real behaviour of the transition by:

$$
E_{r}=\frac{1}{t_{99}-t_{1}} \int_{t_{1}}^{t_{99}} \frac{|C(t)-M(t)|}{|M(t)|} d t
$$

with $t_{1}$ and $t_{99}$ defined as:

$$
\begin{aligned}
& C\left(t_{1}\right)=0.01 \times\left(L_{f}-L_{i}\right)+L_{i} \\
& C\left(t_{99}\right)=0.99 \times\left(L_{f}-L_{i}\right)+L_{i}
\end{aligned}
$$

In spite of the presence of an error on simulated response time due to our polynomial estimation, we obtain a mean relative error $E_{r}$ close to $4 \%$ : for these transitions, our model correctly fits the actual behaviour of the transition.

Furthermore, due to the analogue noise in the signal computed from the measurement system, this relative error can be reduced to $3 \%$ with a more accurate measurement system.

With the response time estimation presented in section 3.1, the LC behaviour in section 3.2, and the response time computation of $\uparrow \mathbf{T}_{*}$ in section 4 , we can estimate the response time for the whole rising transition set. This theoretical response time estimation, shown in figure 9, represents $\frac{256 \times 256-256}{2}$ values (or 32640) for a display panel with 8 bit per colour components.

Our response time estimation is well-correlated with the measurements when $p=\frac{L_{i}}{L_{f}}$ is not close to 1 or when $L_{i}=0$. Actually, we obtain a mean response time estimation error around $5 \%$ when $\frac{L_{i}}{L_{f}}<0.8$ or $L_{i}=0$, which represents more than $80 \%$ of the possible rising transitions.

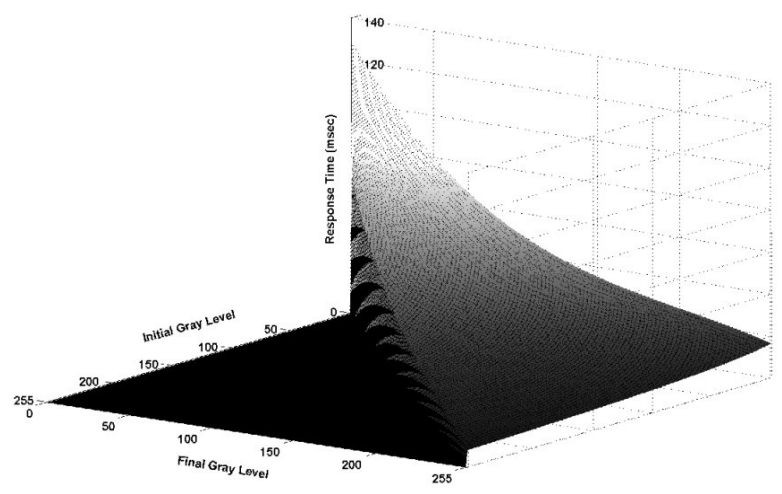

Figure 9: Response time estimation results for 32640 rising transitions.

The case $\frac{L_{i}}{L_{f}}$ close to 1 represents very small rising transitions with a short response time. The obtained results differ slightly from the measurements with a mean relative error close to $10 \%$. In fact, in this case, a slight absolute error on response time estimation implies a high relative error on the final results.

Nevertheless, these relative errors can be drastically decreased by a more accurate measurement system with less analogue noise on the signal.

\begin{tabular}{|c|c|}
\hline Step description & $\begin{array}{c}\text { Mean relative } \\
\text { error }\end{array}$ \\
\hline $\begin{array}{c}\text { Simulated response time } \\
\text { the set } \uparrow \mathbf{T}_{0}\end{array}$ & $<5 \%$ \\
\hline $\begin{array}{c}\text { Curve fitting for very small } \\
\text { transitions from the set } \uparrow \mathbf{T}_{*}\end{array}$ & $7-10 \%$ \\
\hline $\begin{array}{c}\text { Curve fitting for other transitions } \\
\text { from } \uparrow \mathbf{T}_{*}\end{array}$ & $3-5 \%$ \\
\hline $\begin{array}{c}\text { Final LCD response time } \\
\text { estimation }\end{array}$ & $\mathbf{7 \%}$ \\
\hline
\end{tabular}

Table I: Summary of the mean relative error on each model step.

Finally, Table I sums up all the different steps presented in this article and associates their mean relative error. We can notice that the mean relative error of the final LCD response time estimation for rising transitions is around $7 \%$ with only 7 mesaurements.

In our model description, relative errors are cumulative: a slight error on simulated response time implies errors on the curve fitting for transitions of $\uparrow \mathbf{T}_{0}$ and $\uparrow \mathbf{T}_{*}$. Consequently, the more the amount of 
measurements $m$ increases, the more the simulated response time is accurate and the more the mean relative error of the final response time estimation decreases.

The main purpose of our model is to speed up the generation of L.U.T. overdrive data. In fact, filling-up half of the $32 \times 32$ overdrive L.U.T. (corresponding to rising transition correction values) needs 496 response time measurements while with our model, only 7 measurements are required. Indeed, we save more than $95 \%$ of the time to take measurements.

\section{Conclusion}

In this paper, we proposed a general model to describe LCD response time behaviour. This novel approach permits to determine the LCD response time through a simplified set of parameters and all the grayto-gray response times can be easily obtained from a reduced measurement set.

Finally, this model can help all the applications that need to gather all the response times (like overdrive) in order to reduce the computation time for filling-up a Look-Up-Table.

In the future, we will conduct a more detailed investigation on generalized response time estimation that will be applicable to the most LC Display types and we will develop a process to extract from our model the accurate overshoot values for the overdrive application.

\section{References}

1 J. Miseli, "Motion artifacts", SID Symposium Digest of technical papers, vol. 35, pp86-89, 2004.

2 T. Nose, M. Suzuki, D. Saski, M. Imai, and H. Hayama,"A black stripe driving scheme for displaying motion pictures on LCDs", SID Symposium Digest of technical papers, vol. 32, pp. 994-997, 2001.

3 N. Fisekovic, T.Nauta, et al., "Improved motionpicture quality of AM-LCDs using scanning backlight," Asia Display /IDW 01, pp. 1637-1640, 2001.

4 G. Itoh and M. Mishima, "Novel frame interpolation method for high image quality LCDs," Asia Display /IDW $04,2004$.

5 M.-A. Klompenhouwer and L.-J. Jeong, "Motion blur reduction for liquid crystal displays: Motion compensated inverse filtering," Proc. SPIE-IS\&T Electronic Imaging, vol. 5308, 2004.

6 H. Okumura et al., "A new low-image-lag drive method for large-size LCD-TVs," Proc. SIS , pp. 601-604, 1992.

7 P. Adam, P. Bertolino and F. Lebowsky, "A simple LCD response time measurement system based on a CCD line camera", Asia Display 07, Shanghai (China), 2007.

8 T. Kim, B. Park, J. Park, B.-H. Berkeley, and S.-S. Kim, "An optimized boost table measurement method for response time acceleration in LCD," SID Symposium Digest of technical papers, vol. 35, pp.
372-375, 2004.

9 B. Park, T. Kim, J. Oh, S.-W. Lee, J. Park, B.-H. Berkeley, and S.-S. Kim, "Automated system for response time optimization and flicker reduction in LCD manufacturing”, SID Symposium Digest of technical papers, vol. 36, pp. 1797-1799, 2005.

10 Video Electronics Standards Association FPDM Task Group, Flat Panel Display Measurements Standards v2.0, May 2005. 